\title{
Decentralized Multicell Coordinated Beamforming with Overlapped Clusters
}

\author{
Pan Chen, Shengqian Han, and Chenyang Yang \\ School of Electronics and Information Engineering, Beihang University, Beijing 100191, China \\ Email: cplinli@ee.buaa.edu.cn, sqhan@ee.buaa.edu.cn, cyyang@buaa.edu.cn
}

\begin{abstract}
This paper studies downlink coordinated beamforming schemes with overlapped clusters, where a base station (BS) may belong to multiple clusters selected by different users. Subject to the inherent constraint on information sharing for overlapped clusters, i.e., the information of a user is shared only within the user's coordinating cluster, we propose two decentralized coordinated beamforming schemes, which can be implemented at every BS individually without the iterations between multiple BSs. Simulation results demonstrate the performance gain of the proposed schemes over existing distributed schemes.
\end{abstract}

\section{INTRODUCTION}

To meet the 1000x increase in mobile traffic load over the next decade, various inter-cell interference (ICI) management techniques have been studied to fully utilize the scarce spectrum resource, among which coordinated multi-point (CoMP) transmission has attracted much attention [1]. CoMP forms coordinating clusters and then mitigates the ICI within each cluster through joint processing or coordinated beamforming.

Considering the practical constraints on system complexity and signalling overhead, a CoMP cluster usually consists of a limited number of base stations (BSs). The clusters in the network can be non-overlapped or overlapped, depending on the employed clustering methods. BS-centric clustering approaches usually produce non-overlapped CoMP clusters, which can be formed statically by selecting geometrically neighboring BSs [2] or dynamically based on channel state information (CSI) [3]. These approaches are easy for implementation, but the user equipments (UEs) located at the cluster edge still suffer from severe interference from surrounding clusters. This problem can be solved by user-centric clustering, with which UEs may prefer different sets of coordinated BSs, and the resulting clusters are overlapped in general such that a BS may belong to multiple clusters selected by different UEs [4-7].

For CoMP with non-overlapped clusters, the design of coordinated beamforming schemes has been extensively studied in the literature. Depending on what information is shared and where the precoders are computed in a CoMP cluster, we can divide existing schemes into four categories,

This work was supported in part by the National High Technology Research and Development Program of China (No. 2014AA01A705), by the National Natural Science Foundation of China (No. 61301084), and by the Fundamental Research Funds for the Central Universities. centralized, decentralized, iteratively distributed, and noniteratively distributed. Specifically, the centralized schemes suppose that there is a central unit (CU) in each CoMP cluster, which gathers the CSI from all BSs to all UEs in the cluster, and then computes the precoders for all BSs [8]. The decentralized schemes suppose no $\mathrm{CU}$, with which each BS needs to gather the information from all other coordinated BSs and then compute its own precoder [9]. The iteratively distributed schemes assume limited information exchanging among the coordinated BSs, e.g., in [10] the power allocation information is shared, based on which each BS computes its own precoder separately. However, to obtain the final precoder, multi-BS iterations are required in these schemes, i.e., every BS needs to repeatedly update its shared information and compute its precoder according to the updated information from other BSs. The non-iteratively distributed schemes do not require any information exchange among the BSs, with which each BS computes its own precoder only for once [11-13].

For CoMP with overlapped clusters, the precoder design receives relatively little attention. The centralized schemes for joint processing and coordinated beamforming were optimized in [5] and [7], respectively. Since all clusters are overlapped in this case, the CU needs to gather the CSI from all BSs to all UEs in the whole network, which leads to heavy burden in information sharing over the backhaul. Moreover, the centralized schemes jointly optimize the precoders for all BSs in the whole network, which require very high complexity. In [7], an iteratively distributed scheme was proposed where the precoders of all BSs are iteratively updated with the assistance of user feedback. The scheme reduces the information sharing burden over the backhaul compared to the centralized schemes, which however is still of high complexity and has large processing delay due to the multi-BS iterations. The non-iteratively distributed schemes proposed in [11-13] are applicable to overlapped CoMP clusters, which are of low complexity but at the penalty of performance loss because they assume no information exchange among the BSs.

In this paper, we study decentralized coordinated beamforming schemes for overlapped clusters, where each UE receives data only from its master BS. We propose two decentralized schemes, which allow information exchange among the BSs, but each UE's information is only shared within the UE's own cluster. Therefore, the information sharing burden is much less than the centralized schemes. Moreover, 


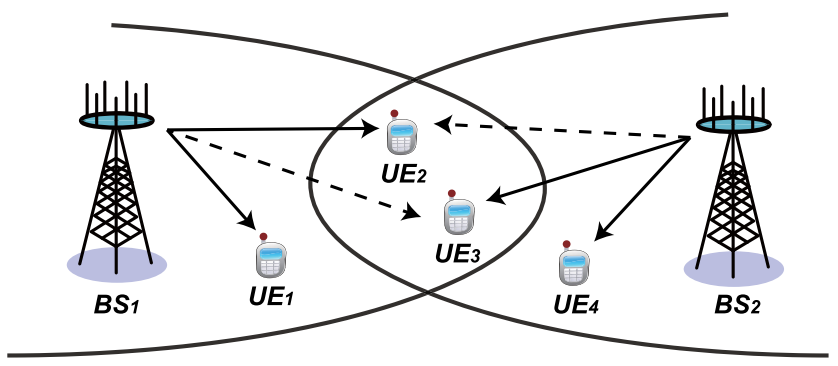

Fig. 1. Illustration of overlapped clusters with $N_{c}=2$ and $K=4$, where the solid and dash arrows indicate the master BS and the coordinated BS for each UE, respectively, $\mathcal{D}_{1}=\{1,2\}, \mathcal{D}_{2}=\{3,4\}, \mathcal{U}_{1}=\{1,2,3\}$, $\mathcal{U}_{2}=\{2,3,4\}, b_{1}=b_{2}=1, b_{3}=b_{4}=2, \mathcal{C}_{1}=\{1\}, \mathcal{C}_{2}=\mathcal{C}_{3}=\{1,2\}$, and $\mathcal{C}_{4}=\{2\}$.

the proposed schemes do not need multi-BS iterations, and hence have much lower complexity and less processing delay than the iteratively distributed schemes. Simulation results demonstrate the performance gain of the proposed schemes over existing non-iteratively distributed schemes.

\section{System Model}

Consider a downlink user-centric CoMP system consisting of $N_{c}$ BSs and $K$ UEs, where each BS is equipped with $N_{t}$ antennas and each UE has a single antenna. We assume that the clusters have been formed with existing approaches, e.g., the method in [6], and focus on the design of coordinated beamformer with overlapped clusters. Denote $\mathcal{U}=\{1, \ldots, K\}$ as the set of all UEs, $\mathcal{D}_{b} \subset \mathcal{U}$ as the set of local UEs receiving data from $\mathrm{BS}_{b}, \mathcal{U}_{b}$ as the set of UEs selecting $\mathrm{BS}_{b}$ as a master or coordinated BS, $\mathcal{D}_{b} \subseteq \mathcal{U}_{b} \subseteq \mathcal{U}, b_{k}$ as the index of the master BS sending data to $\mathrm{UE}_{k}$, and $\mathcal{C}_{k}$ as the set of BSs located in the cluster selected by $\mathrm{UE}_{k}$, where $\mathcal{C}_{k} \subseteq\left\{1, \cdots, N_{c}\right\}$ and $b_{k} \in \mathcal{C}_{k}$. In the cluster for a specific UE (say $\mathrm{UE}_{k}$ ), we suppose that the master $\mathrm{BS}$ of $\mathrm{UE}_{k}, b_{k}$, serves as a $\mathrm{CU}$ in charge of information sharing among the coordinated BSs, beamformer computation, and data transmission. An example of the considered user-centric CoMP network is illustrated in Fig. 1, where $\mathrm{UE}_{1}$ is a local $\mathrm{UE}$ of $\mathrm{BS}_{1}$, or in other words, $\mathrm{BS}_{1}$ is the master $\mathrm{BS}$ of $\mathrm{UE}_{1}, \mathrm{UE}_{3}$ is an other-cell coordinated UE of $\mathrm{BS}_{1}$ that chooses $\mathrm{BS}_{1}$ as a coordinated $\mathrm{BS}$, and conversely $\mathrm{BS}_{1}$ is a coordinated BS selected by $\mathrm{UE}_{3}$.

Let $\mathbf{h}_{k b} \in \mathbb{C}^{N_{t} \times 1}$ denote the channel vector from $\mathrm{BS}_{b}$ to $\mathrm{UE}_{k}$ for $k \in \mathcal{U}$, and $\mathbf{w}_{k b} \in \mathbb{C}^{N_{t} \times 1}$ denote the beamforming vector for $\mathrm{UE}_{k}$ at $\mathrm{BS}_{b}$. In the overlapped clusters, each UE is subject to the intra-cluster interference from its master BS and the coordinated BSs in the selected cluster, and the intercluster interference from the uncoordinated BSs outside of the cluster.

Then we can express the received signal of $\mathrm{UE}_{k}$ as

$$
y_{k}=\underbrace{\mathbf{h}_{k b_{k}}^{H} \mathbf{w}_{k b_{k}} s_{k}}_{\text {Desired signal }}+\underbrace{\sum_{b \in \mathcal{C}_{k}} \sum_{i \in \mathcal{D}_{b}, i \neq k} \mathbf{h}_{k b}^{H} \mathbf{w}_{i b} s_{i}}_{\text {Intra-cluster interference }}
$$

$$
+\underbrace{\sum_{\bar{b} \notin \mathcal{C}_{k}} \sum_{l \in \mathcal{D}_{\bar{b}}} \mathbf{h}_{k \bar{b}}^{H} \mathbf{w}_{l \bar{b}} s_{l}}_{\text {Inter-cluster interference }}+n_{k}
$$

where $s_{k}$ is the data symbol with unit variance destined to $\mathrm{UE}_{k}$, and $n_{k}$ is the additive white Guassian noise with zero mean and variance $\sigma_{k}^{2}$ at $\mathrm{UE}_{k}$.

The signal-to-interference-plus-noise ratio (SINR) at $\mathrm{UE}_{k}$ can be expressed as

$$
\gamma_{k}=\frac{\left|\mathbf{h}_{k b_{k}}^{H} \mathbf{w}_{k b_{k}}\right|^{2}}{I_{k}+\bar{I}_{k}+\sigma_{k}^{2}}
$$

where $I_{k}=\sum_{b \in \mathcal{C}_{k}} \sum_{i \in \mathcal{D}_{b}, i \neq k}\left|\mathbf{h}_{k b}^{H} \mathbf{w}_{i b}\right|^{2}$ is the intra-cluster interference, and $\bar{I}_{k}=\sum_{\bar{b} \notin \mathcal{C}_{k}} \sum_{l \in \mathcal{D}_{\bar{b}}}\left|\mathbf{h}_{k \bar{b}}^{H} \mathbf{w}_{l \bar{b}}\right|^{2}$ is the intercluster interference.

In user-centric CoMP systems, the inter-cluster interference $\bar{I}_{k}$ is controlled by the employed clustering approaches, with which the strong interference can be avoided.

In the paper, we focus on the mitigation of intra-cluster interference $I_{k}$ by designing the information sharing and coordinated beamforming strategy within the cluster formed for $\mathrm{UE}_{k}$. The inter-cluster interference $\bar{I}_{k}$, can be mitigated by judiciously designing user-centric clustering, which however is beyond the scope of the paper.

Since one of the main goals of user-centric clustering is to reduce the information sharing burden on the network and the coordinated complexity, we impose the following two constraints in the design of decentralized coordinated beamforming schemes.

- We consider that each BS has only the CSI from it to its local UEs and the other-cell coordinated UEs that select it as a coordinated $\mathrm{BS}$, rather than assuming the knowledge of the CSI from the BSs to all UEs in the whole network as in $[5,7]$. For example, $\mathrm{BS}_{b}$ only has the CSI from it to $\mathrm{UE}_{k}$ for $k \in \mathcal{U}_{b}$.

- We consider that the information of a UE is shared only within the cluster selected by the UE, rather than among the whole network. For example, the master BS of $\mathrm{UE}_{k}$, $\mathrm{BS}_{b_{k}}$, can only share $\mathrm{UE}_{k}$ 's information to $\mathrm{BS}_{b}$ for $b \in \mathcal{C}_{k}$.

\section{Decentralized Coordinated BeAmForming}

Considering the two constraints stated in Section II, it is non-trivial to study what information should be shared and how to exploit the shared information for precoder design in overlapped clusters. In this section, we first present the CSI sharing based beamformer, which is an extension of the decentralized scheme proposed for non-overlapped clusters in [9]. We use this scheme to analyze the challenges in designing coordinated beamformer with overlapped clusters compared with the non-overlapped clusters.

\section{A. CSI Sharing based Beamformer}

In user-centric CoMP systems, the CSI of a UE is shared only among its selected coordinated BSs. Specifically, if $\mathrm{UE}_{k}$ selects $\mathrm{BS}_{b}$ as its coordinated $\mathrm{BS}$, then $\mathrm{BS}_{b}$ will have the CSI from $\mathrm{BS}_{j}$ to $\mathrm{UE}_{k}$ for all $j \in \mathcal{C}_{k}$. For example, in Fig. 1 with 
CSI sharing, $\mathrm{BS}_{1}$ has $\left\{\mathbf{h}_{11}, \mathbf{h}_{21}, \mathbf{h}_{22}, \mathbf{h}_{31}, \mathbf{h}_{32}\right\}$ and $\mathrm{BS}_{2}$ has $\left\{\mathbf{h}_{21}, \mathbf{h}_{22}, \mathbf{h}_{31}, \mathbf{h}_{32}, \mathbf{h}_{42}\right\}$. One can see that different BSs have different CSI, depending on the clustering results. By contrast, every coordinated BS in a non-overlapped cluster will gather the same CSI from all BSs to all UEs, since the clusters formed for all UEs are the same. This will lead to an incomplete view of the network from each BS's perspective under overlapped clusters. For example, in Fig. $1 \mathrm{BS}_{1}$ is unaware of the existence of $\mathrm{UE}_{4}$. Moreover, a BS knows only the existence of the BSs who share information with it, which is determined by the clustering results.

To describe the incomplete view seen by $\mathrm{BS}_{b}$, let $\mathcal{I}_{b}$ denote the set of BSs that are aware by $\mathrm{BS}_{b}$, which consists of $\mathrm{BS}_{b}$ itself and the BSs that share CSI with $\mathrm{BS}_{b}$, and $\overline{\mathcal{D}}_{b, m} \subseteq \mathcal{D}_{m}$ for $m \in \mathcal{I}_{b}$ denote the set of local UEs receiving data from $\mathrm{BS}_{m}$ that can be seen by $\mathrm{BS}_{b}$.

After gathering the CSI from coordinated BSs, the precoders can be optimized at each BS in a decentralized manner. The basic idea is that each BS needs to first estimate other BS's precoders, in order to make beamformer relatively precise, then design its own precoder. In non-overlapped clusters, as we mentioned above, every BS in the cluster will gather the same CSI from all BSs to all UEs. In this case, each BS can jointly design all BS's precoders as a CU, from which its own precoder is then obtained. It is easy to see that the decentralized scheme can achieve the same performance as the centralized schemes in non-overlapped clusters. In the following, we extend the idea to overlapped clusters by letting each BS regard its incomplete view of the network as complete.

Under the incomplete view of the network, the coordinated beamformer at $\mathrm{BS}_{b}$ in user-centric CoMP systems can be optimized as follows. We define an incomplete network from the viewpoint of $\mathrm{BS}_{b}$. The network consists of $\left|\mathcal{I}_{b}\right|$ BSs, where the $m$-th cell for $m \in \mathcal{I}_{b}$ includes $\left|\overline{\mathcal{D}}_{b, m}\right|$ UEs. With CSI sharing, $\mathrm{BS}_{b}$ gathers the CSI from all BSs to all UEs in the incomplete network, then it acts as a $\mathrm{CU}$ to compute the precoders of all BSs, from which its own precoder can be obtained. Aiming at maximizing the weighted sum rate of all UEs that are aware by $\mathrm{BS}_{b}$, the coordinated beamforming optimization problem at $\mathrm{BS}_{b}$ can be formulated as

$$
\begin{aligned}
& \max _{\left\{\mathbf{w}_{k m}\right\}} \sum_{m \in \mathcal{I}_{b}} \sum_{k \in \overline{\mathcal{D}}_{b, m}} \alpha_{k} \log \left(1+\bar{\gamma}_{k}\right) \\
& \text { s.t. } \sum_{k \in \overline{\mathcal{D}}_{b, m}}\left|\mathbf{w}_{k m}\right|^{2} \leq P_{m}^{\max }, \forall m \in \mathcal{I}_{b},
\end{aligned}
$$

where (3b) is the per-BS power constraint, $P_{m}^{\max }$ is the maximum transmit power of $\mathrm{BS}_{m}, \alpha_{k}$ is the weight of $\mathrm{UE}_{k}$, and $\bar{\gamma}_{k}$ is

$$
\bar{\gamma}_{k}=\frac{\left|\mathbf{h}_{k b_{k}}^{H} \mathbf{w}_{k b_{k}}\right|^{2}}{\sum_{\substack{i \in \overline{\mathcal{D}}_{b, b_{k}} \\ i \neq k}}\left|\mathbf{h}_{k b_{k}}^{H} \mathbf{w}_{i b_{k}}\right|^{2}+\sum_{\substack{m \in \mathcal{I}_{b} \\ m \neq b_{k}}} \sum_{j \in \overline{\mathcal{D}}_{b, m}}\left|\mathbf{h}_{k m}^{H} \mathbf{w}_{j m}\right|^{2}+\sigma_{k}^{2}}
$$

Problem (3) is non-convex, whose globally optimal solution is very hard to find. However, there are methods to find its locally optimal solutions, e.g., by the method proposed in [14]. After solving the problem, $\mathrm{BS}_{b}$ then picks out its own precoder, i.e., $\mathbf{w}_{k b}, k \in \mathcal{D}_{b}$.

In the CSI sharing based beamformer, each BS acts as a $\mathrm{CU}$ and the beamformers of all BSs are optimized, from which the beamforming vectors for its own local UEs are selected out. Since the size of the incomplete network is usually far small than that of the whole network, the CSI sharing based beamformer has much lower complexity than the existing centralized schemes, where the latter require the cooperation of the whole network. However, when compared with the non-iteratively distributed schemes, the complexity of the CSI sharing based scheme is still high, because it involves the joint optimization of the precoders at $\left|\mathcal{I}_{b}\right|$ BSs for $\sum_{m \in \mathcal{I}_{b}}\left|\overline{\mathcal{D}}_{b, m}\right|$ UEs. Therefore, it is desirable to further reduce the complexity.

As discussed before, sharing CSI subject to the two constraints for overlapped clusters will lead to incomplete view of the network at each BS. As a result, each BS is aware of only a part of UEs. For instance, in Fig. $1 \mathrm{UE}_{4}$ cannot be observed by $\mathrm{BS}_{1}$, so that $\mathrm{BS}_{1}$ optimizes its own precoder under the assumption that $\mathrm{BS}_{2}$ only serves $\mathrm{UE}_{3}$, which however is not true. Moreover, sharing CSI will lead to the backhauling burden increasing linearly with the number of antennas at the BSs. Therefore, it is necessary to study more efficient information sharing than CSI sharing for reducing both the backhauling burden and the precoder design complexity.

In next subsection, we will propose a so-called ICI sharing based beamformer to address the issues discussed above.

\section{B. ICI Sharing based Beamformer}

In problem (3), the optimization of the beamforming vectors at multiple coordinated BSs is coupled because the ICI experienced by a UE is determined by other-cell beamformers. Therefore, if a BS has the knowledge of ICI generated by coordinated BSs via information sharing, then the problem can be decoupled.

Based on this observation, we next propose the ICI sharing based beamformer, which is comprised of two aspects. First, to share the ICI information to the coordinated BSs, each BS needs to estimate the ICI generated by itself to all other-cell UEs that select it as a coordinated BS, only based on its local channels, i.e. the channels from it to its local UEs and the other-cell coordinated UEs. Second, with the shared ICI information, each BS optimizes its own beamforming vectors. The two aspects are respectively discussed in the following.

1) ICI Estimation: To estimate the ICI at each BS, we first present the following proposition regarding the optimal beamformer, which can be obtained based on the results in [8].

Proposition 1: The optimal coordinated beamformer, aimed at maximizing the weighted sum rate of all UEs with the assumption of fully sharing all $K$ UEs' CSI among all $N_{c}$ $\mathrm{BSs}$, has the following structure

$$
\mathbf{w}_{k b_{k}}^{*}=\sqrt{p_{k}^{*}} \mathbf{f}_{k b_{k}}^{*},
$$

where $\mathbf{f}_{k b_{k}}^{*}=\frac{\left(\sum_{i \in \mathcal{U}, i \neq k} \alpha_{i} \kappa_{i} \mathbf{h}_{i b_{k}} \mathbf{h}_{i b_{k}}^{H}+\nu_{b_{k}} \mathbf{I}\right)^{-1} \mathbf{h}_{k b_{k}}}{\left\|\left(\sum_{i \in \mathcal{U}, i \neq k} \alpha_{i} \kappa_{i} \mathbf{h}_{i b_{k}} \mathbf{h}_{i b_{k}}^{H}+\nu_{b_{k}} \mathbf{I}\right)^{-1} \mathbf{h}_{k b_{k}}\right\|}$ with the 
parameters $\kappa_{i} \geq 0$ and $\nu_{b_{k}} \geq 0$, and $p_{k}^{*}$ can be obtained as

$$
\left[p_{1}^{*}, \ldots, p_{K}^{*}\right]^{T}=\boldsymbol{\Sigma}^{-1}\left[\sigma_{1}^{2} d_{1}, \ldots, \sigma_{K}^{2} d_{K}\right]^{T},
$$

in which $d_{k}=\alpha_{k} \kappa_{k} \mathbf{h}_{k b_{k}}^{H}\left(\sum_{i \in \mathcal{U}, i \neq k} \alpha_{i} \kappa_{i} \mathbf{h}_{i b_{k}} \mathbf{h}_{i b_{k}}^{H}+\right.$ $\left.\nu_{b_{k}} \mathbf{I}\right)^{-1} \mathbf{h}_{k b_{k}}$, and $\boldsymbol{\Sigma}$ is defined as

$$
[\boldsymbol{\Sigma}]_{k i}= \begin{cases}\left|\mathbf{h}_{k b_{k}}^{H} \mathbf{f}_{k b_{k}}^{*}\right|^{2}, & i=k, i, k \in \mathcal{U} \\ -d_{k}\left|\mathbf{h}_{k b_{i}}^{H} \mathbf{f}_{i b_{i}}^{*}\right|^{2}, & i \neq k, i, k \in \mathcal{U} .\end{cases}
$$

The optimal parameter $\kappa_{k}$ is

$$
\begin{aligned}
\kappa_{k}=\frac{1}{\sum_{i \neq k}\left|\mathbf{h}_{k b_{i}}^{H} \mathbf{w}_{i b_{i}}\right|^{2}+\sigma_{k}^{2}} & \\
& \frac{\left|\mathbf{h}_{k b_{k}}^{H} \mathbf{w}_{k b_{k}}\right|^{2}}{\left|\mathbf{h}_{k b_{k}}^{H} \mathbf{w}_{k b_{k}}\right|^{2}+\sum_{i \neq k}\left|\mathbf{h}_{k b_{i}}^{H} \mathbf{w}_{i b_{i}}\right|^{2}+\sigma_{k}^{2}}
\end{aligned}
$$

The optimal parameter $\nu_{b_{k}}$ satisfies

$$
\nu_{b_{k}} \leq \sum_{i \in \mathcal{D}_{b_{k}}} \alpha_{i} / P_{b_{k}}^{\max }
$$

The optimal beamformer $\mathbf{w}_{k b_{k}}^{*}$ presented in Proposition 1 is governed by the parameters $\left\{\kappa_{k}\right\}_{k \in \mathcal{U}}$ and $\nu_{b_{k}}$. In order to estimate the ICI, we next estimate the optimal beamformer by properly selecting these parameters based on the available CSI at each BS, which can be summarized as follows.

- Considering that the optimal coordinated beamformer that maximizes the weighted sum rate usually leads to effective suppression of intra-cluster interference, we approximate the first item in the right-hand side of (8) as $\frac{1}{\sigma_{k}^{2}}$ and approximate the second item as one for small noises. Therefore, we have

$$
\kappa_{k} \rightarrow \frac{1}{\sigma_{k}^{2}}
$$

- For $\nu_{b_{k}}$, we use its upper bound given by (9) to approximate it, i.e.

$$
\nu_{b_{k}} \rightarrow \frac{\sum_{i \in \mathcal{D}_{b_{k}}} \alpha_{i}}{P_{b_{k}}^{\max }}
$$

- Considering that each BS has only local channels, we set the unknown channels as zeros, i.e.,

$$
\mathbf{h}_{i b} \rightarrow \mathbf{0}, i \notin \mathcal{U}_{b} .
$$

By substituting (10), (11) and (12) into Proposition 1, we can obtain the estimated normalized beamforming vectors and power allocation at $\mathrm{BS}_{b}$, denoted by $\hat{\mathbf{f}}_{k b}$ and $\hat{p}_{k}$ for all $k \in \mathcal{D}_{b}$, respectively. Then the beamformer at $\mathrm{BS}_{b}$ can be estimated as

$$
\hat{\mathbf{w}}_{k b}=\sqrt{c_{b} \hat{p}_{k}} \hat{\mathbf{f}}_{k b},
$$

where $c_{b}=\frac{P_{b}^{\max }}{\sum_{k \in \mathcal{D}_{b} \hat{p}_{k}}}$ ensuring the per-BS power constraint.

With (13), the ICI generated by $\mathrm{BS}_{b}$ to a UE selecting it as a coordinated $\mathrm{BS}$, say $\mathrm{UE}_{i}$, can be estimated as

$$
\hat{I}_{i b}=\sum_{k \in \mathcal{D}_{b}}\left|\mathbf{h}_{i b}^{H} \hat{\mathbf{w}}_{k b}\right|^{2} .
$$

Then $\mathrm{BS}_{b}$ shares the ICI estimate $\hat{I}_{i b}$ to the master BS of $\mathrm{UE}_{i}$. This implies that $\mathrm{BS}_{b}$ shares only a scalar for every UE instead of the CSI, and therefore only $\left|\mathcal{U}_{b}\right|-\left|\mathcal{D}_{b}\right|$ scalars are shared in total. Thus, compared with CSI sharing based scheme, it reduces the backhauling burden.
2) Beamformer Optimization: We next propose a beamformer that is scalable to large network size by exploiting the shared ICI information effectively. The basic idea is that we treat the shared ICI as the worst case of the real ICI, which in fact imposes the following constraints on the beamformer at each $\mathrm{BS}$ (say $\mathrm{BS}_{b}$ )

$$
\sum_{k \in \mathcal{D}_{b}}\left|\mathbf{h}_{i b} \mathbf{w}_{k b}\right|^{2} \leq \hat{I}_{i b}, \quad i \in \mathcal{U}_{b} \backslash \mathcal{D}_{b} .
$$

Considering (15), we can obtain a lower bound of the SINR of $\mathrm{UE}_{k}$ in (2) as

$$
\gamma_{k} \geq \frac{\left|\mathbf{h}_{k b_{k}}^{H} \mathbf{w}_{k b_{k}}\right|^{2}}{\sum_{i \in \mathcal{D}_{b_{k}}, i \neq k}\left|\mathbf{h}_{k b_{k}}^{H} \mathbf{w}_{i b_{k}}\right|^{2}+\hat{I}_{k}+\bar{I}_{k}+\sigma_{k}^{2}} \triangleq \tilde{\gamma}_{k},
$$

where the term $\sum_{i \in \mathcal{D}_{b_{k}}, i \neq k}\left|\mathbf{h}_{k b_{k}}^{H} \mathbf{w}_{i b_{k}}\right|^{2}$ is the intra-cell interuser interference (IUI), and $\hat{I}_{k}=\sum_{j \in \mathcal{C}_{k} \backslash\left\{b_{k}\right\}} \hat{I}_{k j}$ is an upper bound of the intra-cluster ICI.

By replacing $\gamma_{k}$ in the problem (3) with $\tilde{\gamma}_{k}$ and further considering the ICI constraints in (15), we can obtain the beamformer optimization problem at $\mathrm{BS}_{b}$, aimed at maximizing the lower bound of weighted sum rate of all UEs that select $\mathrm{BS}_{b}$ as the master $\mathrm{BS}$.

Although the resulting problem involves joint optimization of the beamformers at multiple BSs, it can be decoupled because the lower bound of SINR, $\tilde{\gamma}_{k}$, depends on only the beamforming vectors of the UEs in the same cell and all the constraints on the beamformers at different BSs are not coupled. Therefore, the beamformer at $\mathrm{BS}_{b}$ can be separately optimized as

$$
\begin{aligned}
\max _{\left\{\mathbf{w}_{k b}\right\}_{k \in \mathcal{D}_{b}}} & \sum_{k \in \mathcal{D}_{b}} \alpha_{k} \log \left(1+\tilde{\gamma}_{k}\right) \\
\text { s.t. } & \sum_{k \in \mathcal{D}_{b}}\left|\mathbf{h}_{i b} \mathbf{w}_{k b}\right|^{2} \leq \hat{I}_{i b}, \quad i \in \mathcal{U}_{b} \backslash \mathcal{D}_{b} \\
& \sum_{k \in \mathcal{D}_{b}}\left|\mathbf{w}_{k b}\right|^{2} \leq P_{b}^{\max } .
\end{aligned}
$$

The problem (17) is a single-cell weighted sum rate maximization problem, which is non-convex due to the nonconvexity of the objective function but can be efficiently solved to obtain a locally optimal solution, e.g., by the method proposed in [14] that exploits the equivalence between data rate and mean square error (MSE).

Remark 1: Compared with existing iteratively distributed schemes that rely on multi-BS iterations, the ICI sharing based beamformer can be implemented at each BS individually, and hence has much lower complexity and less processing delay.

Remark 2: Compared to the CSI sharing based scheme, the ICI sharing based scheme can scale well with the network size. This is because, on one hand, it requires a little amount of information sharing, i.e., one scalar for each UE, which efficiently reduces the traffic burden of backhaul links, and on the other hand, each BS only optimizes the precoders of its own local UEs, which can be implemented distributedly and is independent with the network size.

\section{Simulation Results}

In this section, we evaluate the performance of the proposed CSI sharing based beamformer and ICI sharing based beamformer. In simulations, the optimization problems involved in 


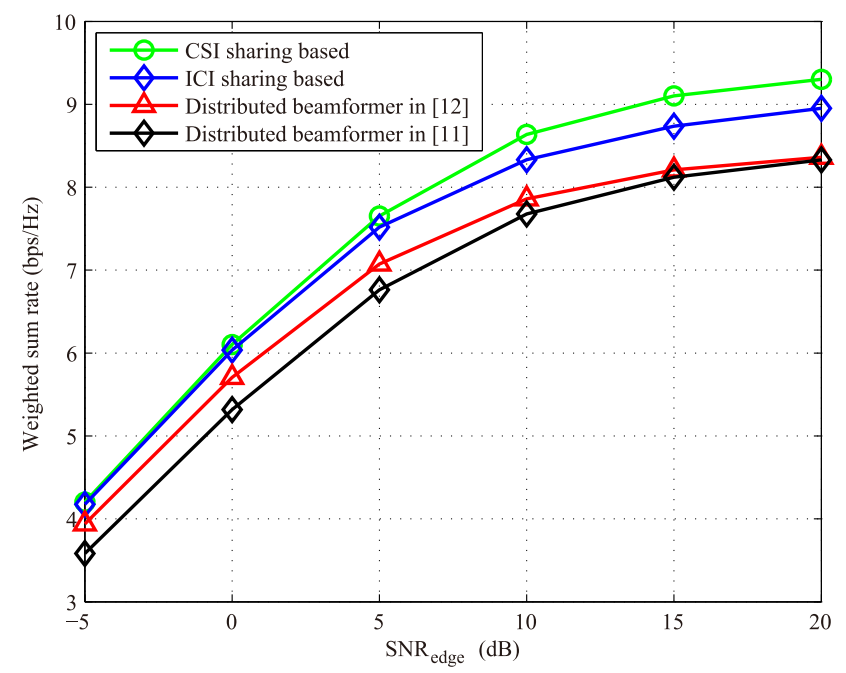

Fig. 2. Weighted sum rate vs. cell-edge SNR.

the two beamformers are solved by the method in [14]. In addition, two existing non-iteratively distributed beamformers proposed in [11] and [12] are also simulated, both of which are the signal-to-leakage-plus-noise ratio (SLNR) based schemes requiring no information exchange among the BSs. Existing centralized and iteratively distributed schemes require information sharing over the whole network, and therefore are not comparable with the proposed schemes.

Considering the complexity in simulation, we simulate a network consisting of $N_{c}=3 \mathrm{BSs}$, where each BS has $N_{t}=2$ antennas and each cell has two randomly placed single-antenna UEs. The cell radius $r$ is set to $250 \mathrm{~m}$. By denoting the average receive signal-to-noise ratio (SNR) of UEs located at the cell edge as $\mathrm{SNR}_{\text {edge }}$, the average receive $\mathrm{SNR}$ of a UE from a BS with distance $d$ can be computed as $\operatorname{SNR}_{\text {edge }}+37.6 \log _{10}\left(\frac{r}{d}\right)$, where $d>50 \mathrm{~m}$. We consider a simple user-centric clustering method, with which a user will select the BSs who have large average channel gains as its coordinated BSs. Specifically, for $\mathrm{UE}_{k}$ we denote the average channel gain from its master BS as $\beta_{k b_{k}}$ in $\mathrm{dB}$; then $\mathrm{BS}_{m}$ will be selected by $\mathrm{UE}_{k}$ if the average channel gain $\beta_{k m}$ from $\mathrm{BS}_{m}$ satisfies $\beta_{k b_{k}}-\beta_{k m} \leq \xi$, where $\xi$ is a pre-determined threshold which is set as $5 \mathrm{~dB}$ in the simulations. As in [8], the weights of UEs are set to $\alpha_{k}=\eta_{b_{k}} / \bar{R}_{k}$ with $\bar{R}_{k}=\mathbb{E}\left\{\log \left(1+\frac{P_{b_{k}}^{\max }}{\left|\mathcal{D}_{b_{k}}\right| \sigma_{k}^{2}}\left|\mathbf{h}_{k b_{k}}\right|^{2}\right)\right\}$, where $\eta_{b_{k}}$ is a scaling factor ensuring $\sum_{i \in \mathcal{D}_{b_{k}}} \alpha_{i}=1$, and $\bar{R}_{k}$ can be regarded as the average data rate of $\mathrm{UE}_{k}$ with equal power allocation. Therefore, the weights reflect the proportional fairness among UEs. The independent and identically distributed (i.i.d.) Rayleigh flat fading channels are considered. All the results are averaged over 100 channel realizations.

The weighted sum rates of the relevant coordinated beamformers are plotted in Fig. 2. We can see that the CSI sharing based beamformer achieves the highest performance at the cost of large information exchange and high complexity for joint optimization of multicell beamformers. The ICI sharing based beamformer performs close to the CSI sharing based beam- former, especially for low and medium SNRs, but requires much less information exchange and complexity. Compared to the two existing non-iteratively distributed beamformers, the proposed beamformers exhibit evident performance gain. The relationship between the relevant beamformers keeps the same when identical weights are considered across the UEs, therefore the results are not shown here.

\section{CONCLUSIONS}

This paper designed decentralized coordinated beamforming with overlapped clusters. We first proposed a CSI sharing based scheme, through which the differences in precoder design between non-overlapped and overlapped clusters were elaborated. We then proposed an ICI sharing based scheme, which requires a little amount of information sharing and low computational complexity, and hence scales well with the network size. Simulation results showed the performance gain of the proposed decentralized schemes over existing distributed schemes.

\section{REFERENCES}

[1] C. Yang, S. Han, X. Hou, and A. F. Molisch, "How do we design CoMP to achieve its promised potential?" IEEE Wireless Commun. Mag., vol. 20, no. 1, pp. 67-74, Feb. 2013.

[2] J. Zhang, R. Chen, J. G. Andrews, A. Ghosh, and R. W. Heath, "Networked MIMO with clustered linear precoding," IEEE Trans. Wireless Commun., vol. 8, no. 4, pp. 1910-1921, Apr. 2009.

[3] A. Papadogiannis, D. Gesbert, and E. Hardouin, "A dynamic clustering approach in wireless networks with multi-cell cooperative processing," in Proc. IEEE ICC, 2008.

[4] Y. Zeng, E. Gunawan, Y. Guan, and J. Liu, "Joint base station selection and linear precoding for cellular networks with multi-cell processing," in Proc. IEEE TENCON, 2010.

[5] C. T. K. Ng and H. Huang, "Linear precoding in cooperative MIMO cellular networks with limited coordination clusters," IEEE J. Select. Areas Commun., vol. 28, no. 9, pp. 1446-1454, Dec. 2010.

[6] J. Gong, S. Zhou, Z. Niu, L. Geng, and M. Zheng, "Joint scheduling and dynamic clustering in downlink cellular networks," in Proc. IEEE GLOBECOM, 2011.

[7] M. Hong, R. Sun, H. Baligh, and Z.-Q. Luo, "Joint base station clustering and beamformer design for partial coordinated transmission in heterogeneous networks," IEEE J. Select. Areas Commun., vol. 31, no. 2, pp. 226-240, Feb. 2013.

[8] S. Han, C. Yang, G. Wang, D. Zhu, and M. Lei, "Coordinated multipoint transmission strategies for TDD systems with non-ideal channel reciprocity," IEEE Trans. Commun., vol. 61, no. 10, pp. 4256-4270, Oct. 2013.

[9] S. Han and C. Yang, "Downlink multicell cooperative transmission with imperfect CSI sharing," in Proc. IEEE ICASSP, 2011.

[10] Y. Huang, G. Zheng, M. Bengtsson, K.-K. Wong, L. Yang, and B. Ottersten, "Distributed multicell beamforming with limited intercell coordination," IEEE Trans. Signal Processing, vol. 59, no. 2, pp. 728738, Feb. 2011.

[11] R. Zakhour, Z. K. M. Ho, and D. Gesbert, "Distributed beamforming coordination in multicell MIMO channels," in Proc. IEEE VTC Spring, 2009.

[12] E. Björnson, N. Jalden, M. Bengtsson, and B. Ottersten, "Optimality properties, distributed strategies, and measurement-based evaluation of coordinated multicell OFDMA transmission," IEEE Trans. Signal Processing, vol. 59, no. 99, pp. 6086-6101, Dec. 2011.

[13] F. Sun and E. de Carvalho, "A leakage-based MMSE beamforming design for a MIMO interference channel," IEEE Signal Processing Lett., vol. 19, no. 6, pp. 368-371, June 2012.

[14] Q. Shi, M. Razaviyayn, Z. Luo, and C. He, "An iteratively weighted MMSE approach to distributed sum-utility maximization for a MIMO interfering broadcast channel," IEEE Trans. Signal Processing, vol. 59, no. 9, pp. 4331-4340, Sept. 2011. 